\title{
Medulloepithelioma (diktyoma) presenting as a perforated, infected eye
}

\author{
MOHAMED A. VIRJI \\ Central Pathology Laboratory, Ministry of Health, Dar-es-Salaam, Tanzania
}

SUMMARY A case of embryonal medulloepithelioma (diktyoma) presenting with perforated infected eye in a 13-year-old Black African girl is described. The tumour mass occupied most of the deformed eye, and invasion of the sclera anteriorly was seen. There was no evidence of orbital or distant tumour involvement. It is suggested that with increasing age these tumours are more likely to show frankly malignant features.

Medulloepithelioma (diktyoma) is a rare neoplasm of the eye which is characterised by slow growth and local invasion and is composed of glandular, neural, and mesenchymal elements (Andersen, 1962). It presents even rarely as an infected perforated eye with a fungating mass replacing the ocular contents. Soudakoff (1936) reported the case of a 28-year-old Chinese who had a perforated eye with tumour mass completely filling it. This paper reports the case of a young black African girl who presented with perforation of one eye and orbital cellulitis initiated probably by a recent rapid growth of a medulloepithelioma (diktyoma) which filled the eye and invaded the cornea.

\section{Case report}

A 14-year-old adolescent girl of the Makonde tribe was seen as a referred case in July 1971 for evaluation and treatment of a probable neoplastic growth in the left eye. There had been complete loss of vision in that eye for three months. The eye had become progressively enlarged, protuberant, and painful and had ruptured spontaneously. Pus discharge had been noticed for a week. The patient was reported to have had poor vision in the left eye since 3 years of age; a white spot had been visible through the pupil; the left eye had been the same size as the right. Until 3 months before admission to hospital there had been no apparent progression of the disease. The family history contained nothing relevant to this case.

Examination showed cellulitis of the left orbit, an

Address for reprints: Department of Laboratory Medicine and Pathology, Medical School, Box 198 Mayo Memorial Building. University of Minnesota, Minneapolis, Minnesota 55455, USA anteriorly perforated left eye, with loss of cornea, purulent discharge, and a fragmenting mass of irregular brownish-grey tissue attached mainly to the superior and temporal portion of the eye and extending into the posterior chamber. The infection was controlled with systemic antibiotics and the left eye was enucleated. Radiological examination of the skull showed no orbital involvement, and chest $x$-rays were normal. Postoperative recovery was uneventful. There was no evidence of tumour recurrence at 6 months, and the patient was subsequently lost to follow-up.

\section{Pathological findings}

The left eye measured $33 \times 27 \times 26 \mathrm{~mm}$. There was a large anterior perforation with loss of cornea, part of conjunctiva, and sclera. Pus and exudate were present on the ragged perforation edges. Bulbar conjunctiva and parts of the sclera were also covered with fibrinous exudate. The eye was deformed and on sectioning showed extensive disorganisation of the contents. A solid fragmenting tumour mass filled the anterior half of the eye (Fig. 1). The tumour was brown to grey, the anterior portion was covered with pus, and some areas felt mucoid. Detached retina occupied part of the posterior portion. On gross examination there was no penetration of the sclera away from the perforation margins.

Microscopically the tumour consisted mainly of an array of glandular structures varying widely in size, shape, and configuration. The gland spaces contained lightly haematoxyphilic hyaline material resembling vitreous (Fig. 2). The glands and interlacing cords were lined with crowded columnar 


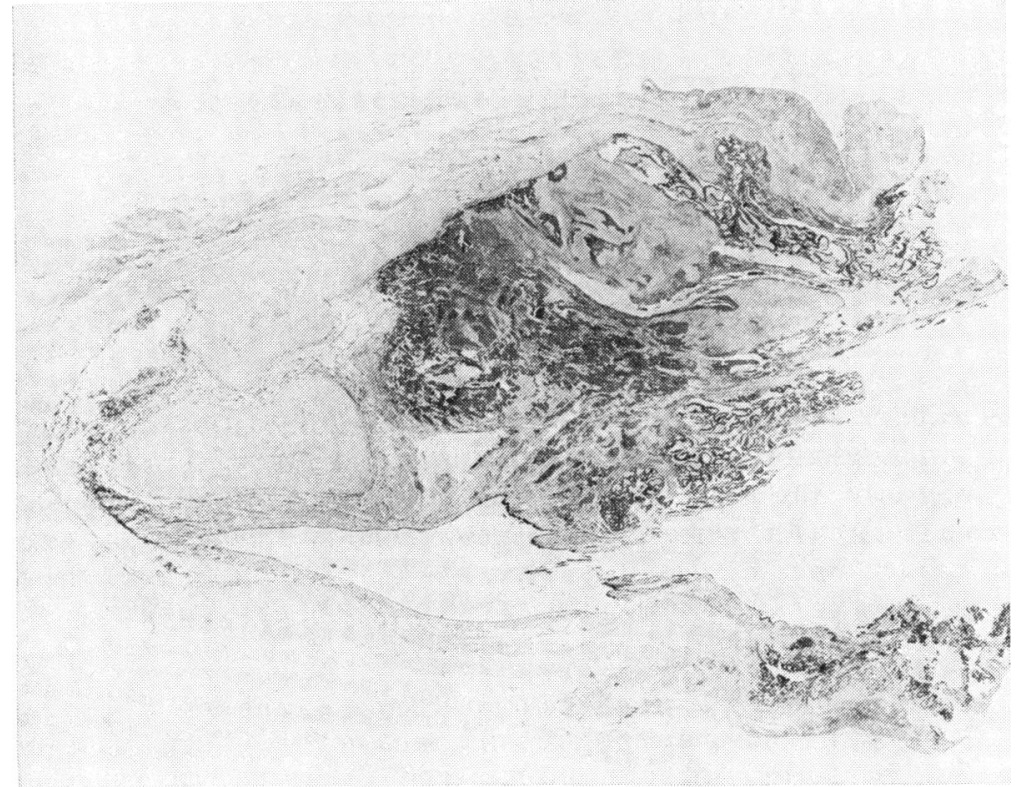

Fig. 1 Section of the whole eye showing the tumour mass, disorganised orbital contents, and the anterior perforation (haematoxylin and eosin, $\times 3$ )

cells with basally placed ovoid hyperchromatic nuclei. Many mitoses were seen, but no abnormal ones were identified. The cells were supported on well defined limiting membrane, and there was little intervening stroma. Cellular debris was seen in the hyaline membrane.

Irregularly interspersed with the glandular tissue were areas containing solid sheets of cells, and in some places the glands seemed to merge with the solid zones. The solid areas consisted partly of polygonal cells with cytological resemblance to the cells forming the glands. There were irregular cleftlike spaces without any hyaline material. At their junction with glandular tissue these cells formed columns merging with the glands. Mitoses were frequent but variable. Clumps of melanin pigment

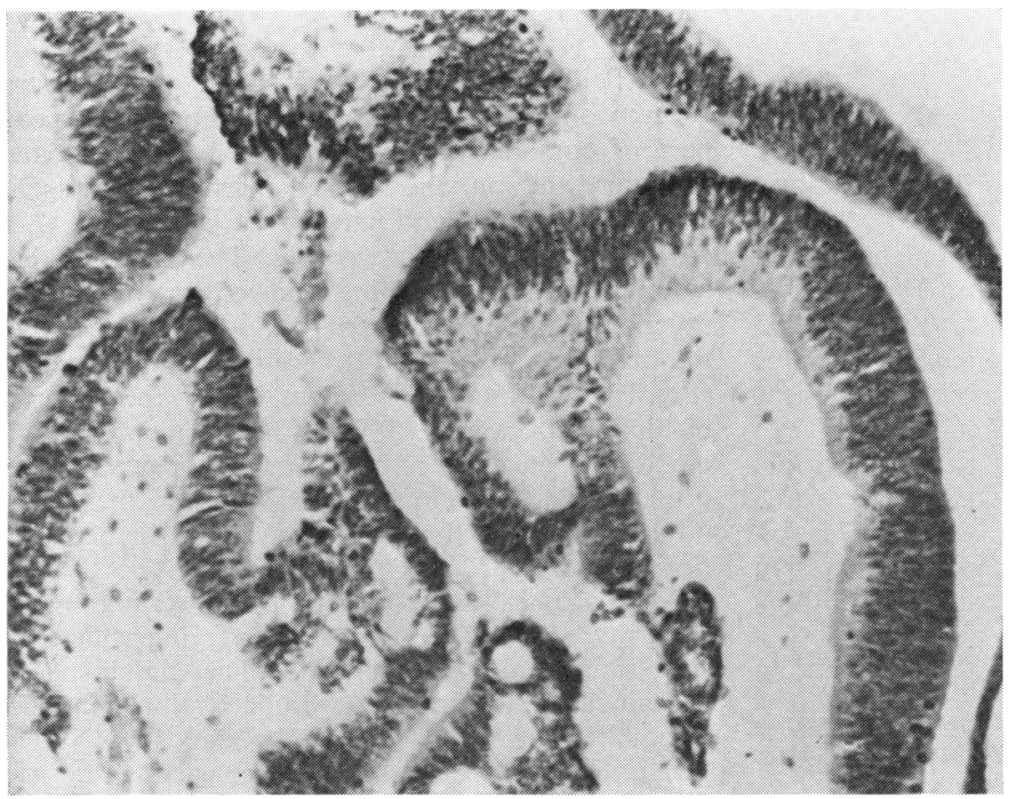

Fig. 2 Glandular spaces of varying size, lined by crowded columnar epithelial cells with limiting membranes. Hyaline material with cell debris fills the spaces (haematoxylin and eosin, $\times 125$ ) 


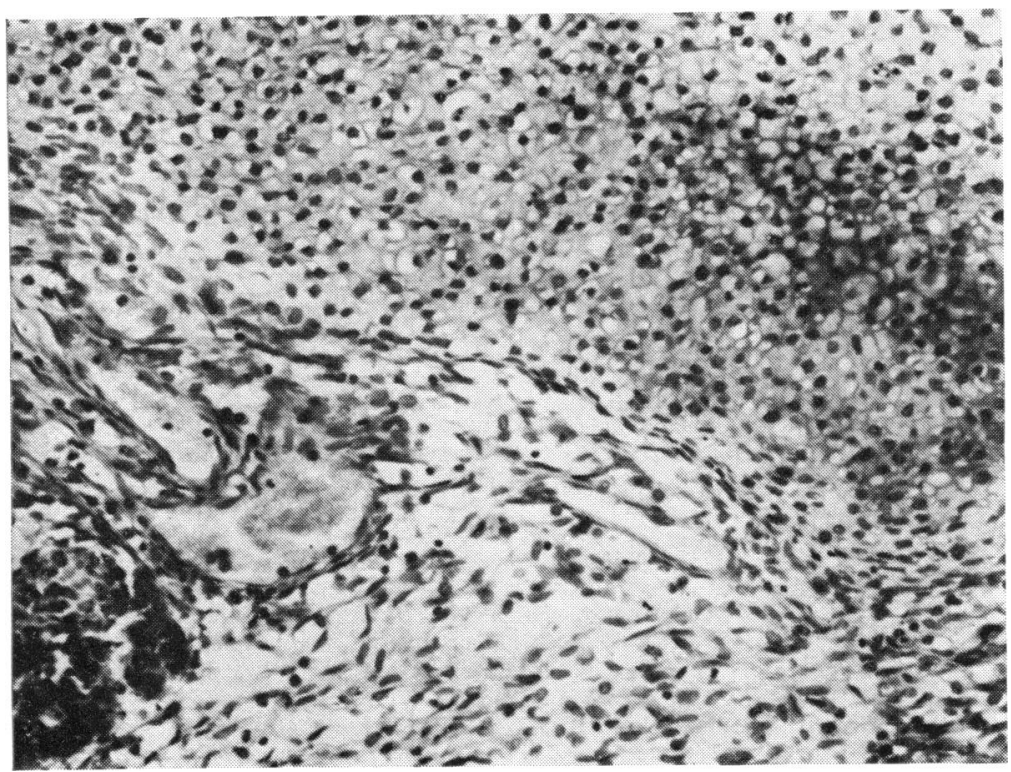

Fig. 3 The spindle cells are seen to merge with the cartilage cells. Sinusoidal spaces surrounded by spindle cells are present in the lower half (haematoxylin and eosin, $\times 350$ )

occurred intercellularly and also in groups of cells, whose cytologic features were obscured by the pigment (Fig. 3). Forming part of the solid areas were spindle cells with ovoid nuclei containing vesicular chromatin. A few sinusoidal spaces were present in these areas and were lined by the spindle cells. Mitoses were rare. In two places there were islands of haematoxyphilic hyaline cartilage. The spindle cells merged with the chondroid elements at the periphery of the islands (Fig. 3). The chondroid cells were uniform in appearance and no mitoses were observed. In several areas there were groups of spongioblasts, which had indistinct cytoplasmic boundaries and rounded, relatively large nuclei with a moderate mitotic activity (Fig. 4). Calcific foci, rhabdomyoblasts, or ganglion cells were not detected. Tissue sections from the perforation margins showed corneal keratinisation and thickening, chronic granulation tissue, and necrotic debris. Glandular tumour tissue could be identified in the partially necrotic areas, and small foci of neoplastic glands were seen in the conjunctival and scleral tissues.

\section{Discussion}

The history in this case indicates that the tumour or an abnormal mass was in the eye from 3 years of age without any noticeable progression until recently. It is, however, not known whether the patient had a progressive loss of vision in the left eye over the years. Histological features are suggestive of active, proliferative neoplastic tissue, and it is probable that the tumour had undergone recent rapid growth after a long initial, relatively quiescent period, with the glandular component exhibiting more aggressive invasive characteristics, leading to perforation of the eye. The nature of the stimulus or stimuli leading to this change is speculative. In the group of embryonal type of medulloepitheliomas reviewed by Andersen (1962) the mean duration of symptoms before diagnosis was 4 months with a range of up to 3 years for benign tumours and a mean duration of 1 year with a range of 2 months to 9 years for malignant tumours. In all these cases the tumours appeared histologically to be at least

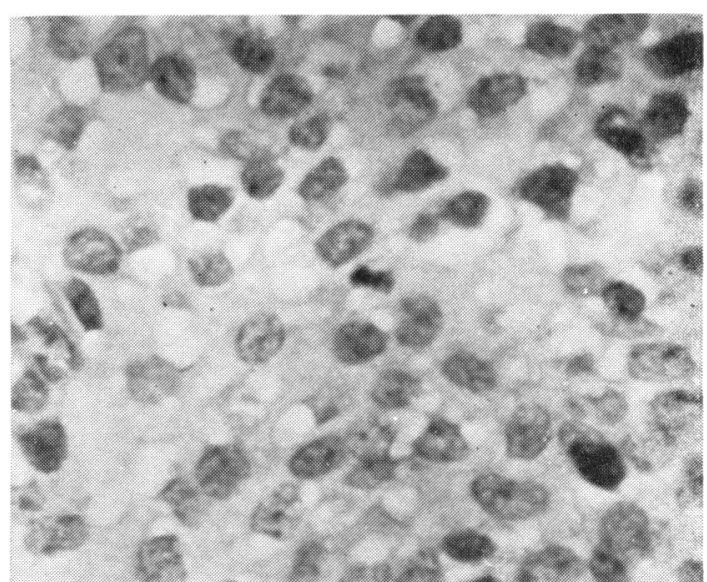

Fig. 4 Spongioblasts with mitotic activity

(haematoxylin and eosin, $\times 500$ ) 
locally invasive, and the malignant ones were so characterised mainly because of extraocular, orbital invasion, pronounced pleomorphism, mitotic activity, and an absence of ganglion cells. The present case had all these features except orbital invasion. Cardell and Starbuck (1959), in reviewing cases of medulloepitheliomas (diktyomas), found that advanced tumours with retinal or scleral involvement presented in older age groups. It is likely therefore that with increasing age the tumour may show characteristics of frank malignancy and that, left alone one, or more of the cellular elements may exhibit features of vigorous growth and tissue invasion extraocularly.

Andersen (1962) has suggested that the chondroid and spindle-cell components are part of stromal reaction to the tumour, possibly in the persistent primary vitreous. Zimmerman et al. (1972) reported 4 cases of medulloepithelioma containing rhabdomyosarcomatous component, and in one of these the recurrent orbital tumour had chondrosarcoma tissue only. They suggested that the primitive medullary epithelium is pluripotential. In the present case the chondroid and spindle cell mesenchymal components did not exhibit pleomorphism or mitotic activity, and no rhabdomyoblasts or ganglioform cells were found. On electron microscopy the ganglioform cells were reported by Zimmerman et al. (1972) to have features of rhabdomyoblasts.

In reviewing a group of orbital tumours in African children in the geographical area in which the patient presented Templeton (1971) did not record any case of embryonal medulloepithelioma. This is believed to be the first such case from the area.

I thank Dr C. Anderson for confirming the diagnosis and discussion of the case.

\section{References}

Andersen, S. R. (1962). Tumors of the eye and adnexa. International Ophthalmology Clinics, 2, 483.

Cardell, B. S., and Starbuck, M. J. (1959). British Journal of Ophthalmology, 43, 217.

Soudakoff, P. S. (1936). Archives of Ophthalmology, 15, 680.

Templeton, A. C. (1971). British Journal of Ophthalmology, 55, 254.

Zimmerman, L. E., Font, R. L., and Andersen, S. R. (1972). Cancer, 30, 817. 